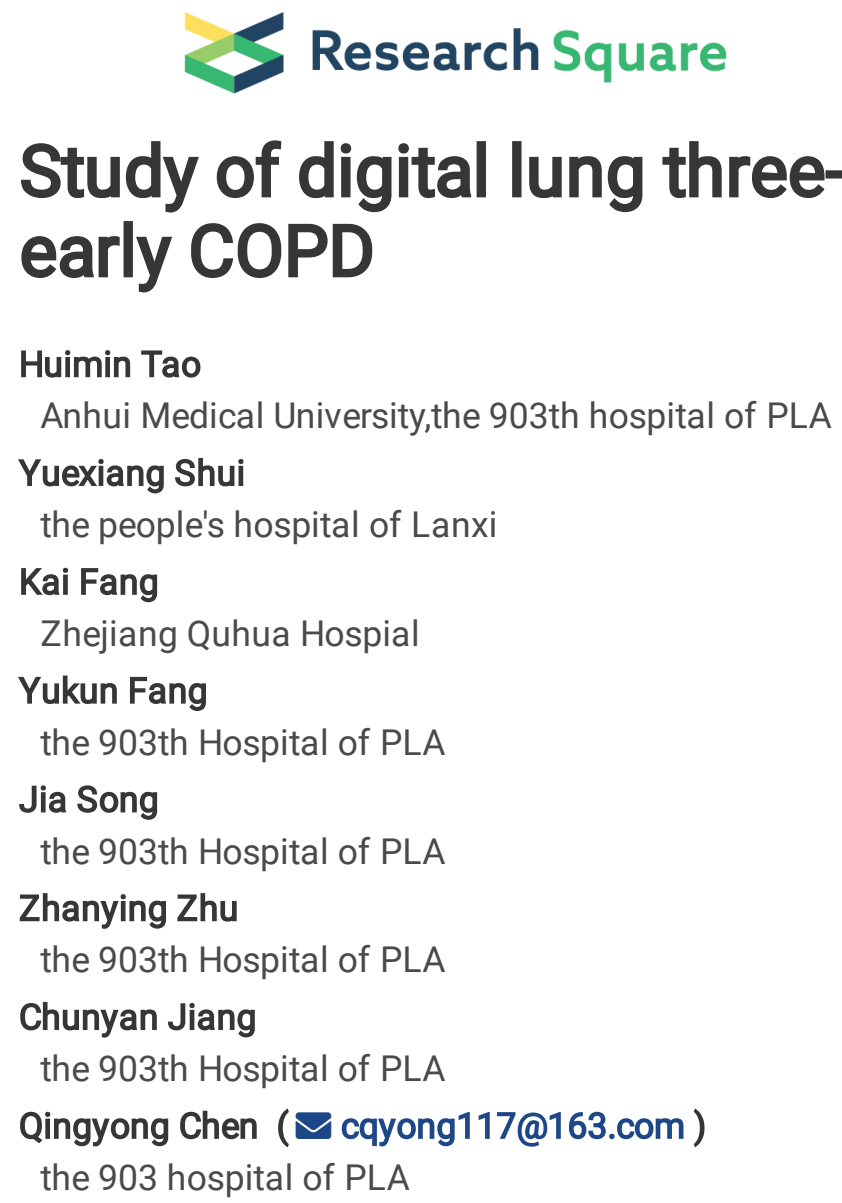

Huimin Tao

Anhui Medical University,the 903th hospital of PLA

\section{Yuexiang Shui}

the people's hospital of Lanxi

Kai Fang

Zhejiang Quhua Hospial

\section{Yukun Fang}

the 903th Hospital of PLA

Jia Song

the 903th Hospital of PLA

Zhanying Zhu

the 903th Hospital of PLA

Chunyan Jiang

the 903th Hospital of PLA

Qingyong Chen ( $\nabla$ cqyong117@163.com )

the 903 hospital of PLA

\title{
Study of digital lung three-dimensional imaging technology in early COPD
}

\section{Research article}

Keywords: COPD, digital lung 3D imaging technology, PFT, small airway disease

Posted Date: July 29th, 2020

DOI: https://doi.org/10.21203/rs.3.rs-49295/v1

License: (1) This work is licensed under a Creative Commons Attribution 4.0 International License. Read Full License 


\section{Abstract \\ Background}

COPD is characterized by progressive development of airflow limitation. Small airway functional and structural disease is considered pivotal in the pathology of early COPD. We used pulmonary function (PFT) and digital lung three-dimensional (3D) imaging technology to detect small airway functional and structural disease that applied for screening early COPD.

\section{Methods}

136 patients underwent PFT and CT at the 903th Hospital of People Liberation Army from January 2018 to January 2019 , including 90 in the observation group and 46 in the control group. We tested the associations between digital lung 3D imaging technology parameters and PFT. Then, we detected small airway functional and structural disease by comparing digital lung 3D imaging technology parameters and PFT between the observation and control groups.

\section{Results}

There were correlations between digital lung 3D imaging technology parameters and PFT. Furthermore, compared with generation 5 bronchus, there were significant correlations between FEV1/FVC with generation 7 WT $(r=-0.592 ; p<0.01)$, and TDR $(r=-0.608 ; p<0.01)$. There were significant correlations between MEF50 with generation 7 WT $(r=-0.607 ; p<0.01)$, LA $(r=0.632 ; p<0.01)$, and WA\% $(r=-0.643 ; p<0.01)$. We found that we could evaluate the lung function change by the technology, and generation 7 bronchus were more effective in assessing small airway disease. There were significant differences between the observation and control groups in digital lung 3D imaging technology parameters and PFT $(P<$ 0.05).

\section{Conclusion}

We found that the observation group had small airway functional and structural disease as compared with control group, which was diagnosed as early COPD. Our results showed that we could use PFT and digital lung 3D imaging technology to detect small airway functional and structural disease that applied for screening early COPD.

\section{Background}

With the increase of air pollution, the incidence of chronic obstructive pulmonary disease (COPD) in China's $\geq 40$-year-old population has increased year by year in 1990-2014 [1]. The 2018 GOLD suggest that COPD is a chronic disease with persistent airflow limitation, and currently the third leading cause of death worldwide [2].

Early COPD is mainly small airway functional and structural disease. Hogg et al. had showed that small airway disease may precede the onset of emphysema. Therefore, it is the key role in COPD [3]. Moreover, lung function damage of early COPD progress rapidly, but it can effectively slow the disease progression after drug treatment $[4,5]$. Advanced COPD is mainly characterized by irreversible airflow limitation, especially in acute exacerbation. It will increase clinical symptoms and has a substantial healthcare cost with high numbers of hospital admissions [6]. Therefore, how to increase the discovery rate of early COPD and guide individualized treatment is essential for improving the prognosis of COPD patients.

Currently, pulmonary function (PFT) is mainly used to screen COPD. The diagnostic criteria of COPD is after inhaling bronchodilator, and the ratio of forced expiratory volume to vital capacity (FEV1/FVC) $<0.70$ in the first second. However, lung function abnormalities occur when more than $30 \%$ of lung tissue damage, so PFT alone may miss diagnosis of early 
disease status [7]. The small airway is difficult to assess, due to airway diameter and anatomical position in the lung. Therefore, we need more effective tools to diagnose early COPD.

Digital lung three-dimensional (3D) imaging technology has been an emerging technology for COPD pulmonary function assessment in recent years. The technology of semi-automated image assessment can show emphysema distribution and airway structure change clearly by separating the lung parenchyma and airways from the chest wall and surrounding structure. Hisham Taher et al pointed out that lung density, generations 5 to 7 bronchus parameters could effectively evaluate airway disease [8]. Furthermore, Diana E. Litmanovich et al proved that it could perform comprehensive analysis airway disease and lung ventilation function changes in COPD patients, which was a complement of PFT. At the same time, compared with PFT, it is fast, and accurate, which can be repeated secondary measurement [9]. However, digital lung 3D imaging technology had not been fully popularized in the diagnosis of COPD. Whether 3D computed tomography (CT) and PFT can screen early COPD remains unknown.

The aim of this study is to evaluate small airway functional and structural disease in early COPD. Recently, developed digital lung 3D imaging technology has offered the possibility to make these comparisons in detail and in a more sensitive way than using PFT. We used PFT and digital lung 3D imaging technology to detect small airway functional and structural disease that applied for screening early COPD.

\section{Objectives}

The objective of review was to screen early COPD and the details were shown as follows;

1. We explore the relationships between digital lung 3D imaging technology parameters and PFT, and,

2. In order to detect small airway functional and structural disease, we compare digital lung 3D imaging technology parameters and PFT between the observation group and control group.

\section{Methods}

\subsection{Patients}

This was a prospective study of 136 patients at the 903th Hospital of People Liberation Army from January 2018 to January 2019. The patients whose PFT showed that small airway ventilation dysfunction and FEV1/FVC>70\% were grouped into the observation group $(n=90)$, while the patients whose PFT showed normal lung function were grouped into the control group ( $n=46)$. The exclusion criteria were as follows: 1$)<40$ and $>70$ years old; 2 ) pregnant woman; 3 ) comorbid lung diseases such as lung cancer, pneumonia, active pulmonary tuberculosis, pulmonary embolism, or interstitilung disease; 4) previous lung surgery; 5) unable to complete PFT; 6) asthma, severe heart, liver, or kidney dysfunction; or 7) CT quality was insufficient for analysis. The study has been approved by the Ethics Committee of the 903th Hospital of People Liberation Army, and all participants gave informed consent.

\subsection{Data collection}

After enrollment, age, sex ratio, smoking composition ratio, body mass index (BMI) and the number of patients were collected.

\subsection{PFT}

The PFT (Master Screen, Jaeger, Germany) was performed. Maximum expiratory flow-volume curve, FVC, pulmonary diffusion function in one breath, and bronchial diastolic function were evaluated after administration of $400 \mu \mathrm{g}$ of salbutamol. The procedure was performed according to the American Thoracic Society/European Respiratory Society guidelines. PFT parameters: forced expiratory volume in the first second (FEV1), measured and predicted percentage of 
forced expiratory volume in the first second (FEV1\%), mid-expiratory flow rate (MEF25-75), 25\%, 50\% and 75\% of maximum expiratory flow (MEF25, MEF50, MEF75), maximum expiratory flow rate (PEF), carbon monoxide diffusion (DLCO), and FEV1/FVC.

\subsection{CT scanning}

Respiratory biphasic CT examinations were performed using a 64-detector CT (Somatom Definition AS; Siemens) with subjects holding their breath at full inspiration in the supine position. Tube voltage was $120 \mathrm{kV}$, and tube current varied by automatic methods based on slice location and participants' body habitus. The exposure time was 0.5 second, and the matrix size was $512 \times 512$ pixels. Images were contiguously reconstructed with a $1 \mathrm{~mm}$ slice thickness (with $0.625 \mathrm{~mm}$ overlapping), using a standard kernel algorithm.

\subsection{Digital lung 3D imaging technology}

The FACT-Digital lung TM software (DeXin, Xi'an, China) was used to perform semi-automated 3D CT quantitative measurements of the bronchial tree (Figure 1). The following three steps were taken. First, the skeleton extraction algorithm was used to perform a 3D bronchial segmentation. The skeletons of all tested bronchial generations were automatically extracted and exhibited as a bronchial tree (Figure 1 (a-b)). Second, virtual bronchoscopy was used to synchronously display the cursor position on the axial, coronal, and sagittal images and the bronchial straightened image (Figure 1 (c-f)). The enlarged axial view of the bronchus (Figure 1 (e)) was synchronously displayed along with measurements, including wall thickness (WT), lumen diameter (LD), thickness to diameter ratio (TDR), WA\% [WA/(WA+LA) $\times 100 \%]$, lumen area (LA) (Figure 1 (f)). Third, the average values of the measurements of all points for a bronchial generation. The measured bronchi were generations 5 to 7 of the right upper lobe tip segment (RB1), the left upper lobe tip segment (LB1), the right lower lobe posterior basal segment (RB10), the left lower lobe posterior basal segment (LB10), and the measurement parameters were WT, LD, TDR, WA\%, LA. In addition, emphysema was quantified by using the percent of lung voxels with density less than -950 Hounsfield Units (HU) (\%LAA950), emphysema area (PRM Emph), lung volume (LV) (Figure 2 (a-b)). We used parametric response mapping to match inspiratory and expiratory images voxel-to-voxel, and calculated the percentage of non-emphysematous gas trapping, or small airway lesion area (PRM fSAD), a measure of small airways disease (Figure 2 $(c-d))$.

\subsection{Statistical analysis}

All data were expressed as ' $x \pm$ SD. Pearson correlation coefficient $(r)$ describes the degree of linear correlation, which is between -1 and 1 . When $r$ is less than -0.5 or greater than 0.5 , it is believable that there is strong negative or positive correlation between two variables. Statistical significance between the two groups was evaluated via studentçs $t$-test. When $P<0.05$, the difference was statistically significant.

\section{Result}

\subsection{Correlations between digital lung 3D imaging technology parameters and PFT}

There were negative correlations between PRM fSAD, PRM Emph, LV, \%LAA950, and WT, TDR, WA\% of generations 5 to 7 bronchial and FEV1, FEV1\%, FEV1/FVC, MEF25-75, MEF25, MEF50, MEF75, PEF $(P<0.05)$. There were negative correlations between PRM Emph, LV, \%LAA950, generation 5 TDR, WA\%, generation 6 TDR, WA\%, generation 7 WA\% and DLCO $(P<$ 0.05). There were positive correlations between LD, LA of generations 5 to 7 bronchial and FEV1, FEV1\%, FEV1/FVC, MEF2575, MEF25, MEF50, MEF75, PEF and DLCO $(P<0.05)$. Among them, compared with generation 5 bronchus, there were significant correlations between FEV1/FVC with generation 7 WT $(r=-0.592 ; p<0.01)$, and TDR $(r=-0.608 ; p<0.01)$. There were significant correlations between MEF50 with generation 7 WT $(r=-0.607 ; p<0.01)$, LA $(r=0.632 ; p<0.01)$, and WA\% ( $r$ $=-0.643 ; p<0.01)$, as shown in Table 1 . 


\subsection{Comparisons of PFT and digital lung 3D imaging technology parameters between the observation group and control group}

As shown in Table 2, there were no significant differences between the two groups in age, sex ratio, smoking composition ratio and $\mathrm{BMI}(P>0.05)$. There were significant differences between the two groups in PFT and digital lung 3D imaging technology parameters (FEV1, FEV1\%, FEV1/FVC, MEF25-75, MEF25, MEF50, MEF75, PEF, DLCO, PRM fSAD, PRM Emph, LV, \%LAA950, and WT, TDR, WA\%, LD, LA of generations 5 to 7 bronchial $(P<0.05))$.

\subsection{Comparison of respiratory biphasic bronchial tree between the two groups}

Compared with the control group, there were small airway structural disease in the respiratory biphasic bronchial tree, especially in the expiratory phase, as shown in Figure 3.

\section{Discussion}

\subsection{Status of early COPD}

COPD is a serious chronic disease, and is associated with increased morbidity and mortality. It is complex, heterogeneous disease characterized by progressive development of airflow limitation, which has become a major public health problem. Rennard SI et al proposed that early COPD was a period before the disease occurs: the disease had not yet produced all the clinical effects. Early COPD mainly had small airway functional and structural disease, which was different from the early stage of COPD [10]. Small airway functional disease is small airway mild airflow obstruction. Structural disease is small airway chronic inflammation, which causes mild small airway wall thickening [11]. Furthermore, lung function damage progress rapidly in early COPD, but it can slow down the disease progression after drug treatment. Therefore, how to improve the diagnosis of early COPD and guide individualized treatment is important.

\subsection{The diagnosis of early COPD has controversial}

In recent years, the diagnosis of early COPD has been controversial at home and abroad. Fernando $\mathrm{J}$ et al proposed early COPD should be studied in those younger than 50 years with 10 or more pack-years smoking history and any of these abnormalities: 1) early airflow limitation (post-bronchodilator FEV1/FVC, lower limit of normal), 2) compatible CT abnormalities, 3) rapid decline in FEV1 (> $60 \mathrm{ml} / \mathrm{yr}$ ) that is accelerated relative to FVC " to define early COPD [12]. However, some people has suggested different views. Alvar Agusti et al found that many patients who no history of smoking and young age also had limited ventilation function, or clinical symptoms [13]. Moreover, there is no clear evidence of a rapid decline in lung function in these patients.

\subsection{Using digital lung 3D imaging technology to resolve disputes}

Now, PFT is the gold standard for the diagnosis of COPD. Small airway functional disease is detected when PFT shows small airway ventilation dysfunction. However, PFT provides little information about structural disease and is poorly related to respiratory symptoms. These findings point to the lack of sensitivity of PFT in detecting small airway structural disease. So it is necessary to develop novel metrics for the detection of early COPD. We hypothesized that the relationship between digital lung 3D imaging technology with small airway structural disease is utilized to diagnose early COPD.

Digital lung 3D imaging technology is a computer-aided diagnosis system, which is a means of assessment of airway disease. Miranda Kirby and Surya P. Bhatt proposed the following functions: 1) evaluate the lung function changes, 2) measure the pathological damages of COPD (peripheral airway stenosis, airflow limitation, etc), 3) reconstruct 3D lung structure, respiratory biphasic bronchial tree $[14,15]$. Digital lung 3D imaging technology has obvious advantages in early COPD screening. Therefore, we investigated small airway structural, functional changes and early COPD by combining digital lung 3D imaging technology with PFT, which has never been reported before. 


\subsection{Selection of parameters and analysis of results}

Chronic inflammation of early COPD is mainly small airway disease, which is an airway with a lumen diameter of $\leq 2 \mathrm{~mm}$, mainly including bronchioles and terminal bronchus. Yan Li et al reported that WT, TDR, WA\%, LD, and LA values of generations 5 to 7 bronchus could effectively evaluate airway disease [16]. The study detected small airways structural disease by the respiratory biphasic generation 5 to 7 bronchi of these parameters. The generation 7 is about $2 \mathrm{~mm}$, which is close to the small airway. At the same time, RB1, LB1, RB10, and LB10 were equivalent to the overall evaluation of the entire lung, which would avoid the difference in respiratory motion.

Some studies reported that distal bronchial parameters were more closely related to small airway airflow limitation than the proximal bronchial in COPD patients [17]. Furthermore, WT and LAA950\% were well-accepted indicators reflecting the pathological changes of COPD. Hogg JC et al reported that FEV1, FEV1\%, FEV1/FVC prompted lung ventilation, small airway inflammation, and exudation in patients, which were closely related with airflow limitation [18]. In the study, compared with generation 5 bronchus, there were significant correlations between FEV1/FVC with generation 7 WT ( $r=$ -0.592; $p<0.01$, which was consistent with the study by Yan Li et al [19]. Moreover, Guangqin Xia et al reported that MEF2575, MEF25, MEF50, MEF75, PEF were consistent with small airway disease [20]. In the study, compared with generation 5, there were significant correlations between MEF50 with generation 7 WT $(r=-0.607 ; p<0.01)$, LA $(r=0.632 ; p<0.01)$, and WA\% $(r=-0.643 ; p<0.01)$. Our results showed that we could evaluate small airway disease by digital lung $3 \mathrm{D}$ imaging technology, and generation 7 bronchus was more effective in assessing small airway disease.

Vasilescu D et al proposed that PRM was a specific density threshold, and a relatively stable indicator for evaluating small airway disease [21, 22]. In this study, the digital lung 3D imaging technology parameters (\%LAA950, PRM fsad et al) and PFT were correlated, which indicated that we could effectively evaluate small airway disease using the technology. In addition, Harvey BG and other studies had found that normal smokers with decreased DLCO also develop COPD, which was consistent with results of the study [23].

\subsection{Assessing small airway functional and structural disease by PFT and digital lung 3D imaging technology in the observation group}

Compared with the control group, there were significant differences between digital lung 3D imaging technology parameters and PFT in the observation group $(P<0.05)$. Furthermore, the respiratory biphasic bronchial tree also showed small airway structural change. Our results showed that the observation group patients had structural and function disease by digital lung 3D imaging technology parameters and PFT, and would be diagnosed as early COPD. The study was an exploration of the diagnosis of early COPD. These functional and structural disease need inclusion in cohort studies of established COPD and early disease, to better understand their clinical relevance in COPD.

\subsection{Limitations}

The current study is not without limitations. Due to the high technical requirements and large data workload, the sample collection was still small. Moreover, we showed that small airway disease patients was compared with the normal. In the future, it will be compared with mild, moderate, severe, very severe COPD, for assessing small airway disease. We will continue to explore the study of early COPD and individualized treatment, and provide more evidence for slowing the progress of the disease.

\section{Conclusion}

Our results showed that we could use PFT and digital lung 3D imaging technology to detect small airway functional and structural disease that applied for screening early COPD. 


\section{Declarations}

\section{Ethics approval and consent to participate}

This study was approved by the ethics committee at the 903th Hospital of People Liberation Army and was performed in accordance with the ethical standards laid down in the Declaration of Helsinki. Written informed consent was obtained from each participant enrolled in the study.

\section{Consent to publish}

All individual materials such private images have been obtained the informed consent before published.

\section{Availability of data and materials}

The datasets and related materials within the above study can be available from the corresponding author on a reasonable request.

\section{Competing interests}

The authors declare that they have no competing interests.

\section{Funding}

This research did not receive any specific grant from funding agencies in the public, commercial, or not-for-profit sectors.

\section{Authors' contributions}

QYC, HMT, CYJ designed and performed the whole study, discussed and analyzed the findings, and wrote the paper. YQS, KF performed a part of the study, discussed and analyzed a part of the findings, and helped write the paper. YKF, JS collected data and discussed the findings. ZYZ helped analyze digital lung 3D imaging. All authors read and approved the final manuscript.

\section{Acknowledgements}

We are very grateful to the 903th Hospital of People Liberation Army and Shenzhou Dexin Company for providing technical support.

\section{References}

1. Chen RC, Zhao DX. Pay attention to the disease burden and patient characteristics of chronic obstructive pulmonary disease in China. Chinese Journal of Medicine. 2017;97(40):3121-3.

2. Agusti AG, Vogelmeier C, Celli BR, et al. Global Strategy for the Diagnosis, Management, and Prevention of Chronic Obstructive Lung Disease 2018 Report. The American Thoracic Society. 2018;27(1).

3. McDonough JE, Yuan R, Suzuki M, et al. Small-airway obstruction and emphysema in chronic obstructive pulmonary disease. N Engl J Med. 2011;365(17):1567-75.

4. McDonough JE, Yuan R, Suzuki M, et al. Small-Airway Obstruction and Emphysema in Chronic Obstructive Pulmonary Disease. The New England Journal of Medicine. 2018;18(8).

5. Koehler U, Hildebrandt O, Koehler N, et al. Are Small Airways the Key to Understanding Pathophysiology and Treatment Efficacy in Chronic Obstructive Pulmonary Diseases? Pneumologie. 2018;72:790-6.

6. United States 
Wheaton AG, Cunningham TJ, Ford ES, et al. Employment and activity limitations among adults with chronic obstructive pulmonary disease - United States. 2013, Morbidity and Mortality Weekly Report (MMWR).

2015;64(11):289-295.

7. Haraguchi M, Shimura S, Hida W, et al. Pulmonary function and regional distribution of emphysema as determined by high-resolution computed tomography. Respiration. 1998;65(2):125-9.

8. Taher H, Bauer $\mathrm{C}$, Abston $\mathrm{E}$, et al. Chest wall strapping increases expiratory airflow and detectable airway segments in computer tomographic scans of normal and obstructed lungs. J Appl Physiol. 2018;124:1186-93.

9. Litmanovich DE, Hartwick K, Silva M. Multidetector Computed Tomographic Imaging in Chronic Obstructive Pulmonary Disease:Emphysema and Airways Assessment. Radiol Clin N Am. 2014;52:137-54.

10. Rennard SI, Drummond MB. Early chronic obstructive pulmonary disease: definition, assessment, and prevention. Lancet. 2015;385:1778-88.

11. Woodruff PG, Barr RG, Bleecker E, et al. Clinical significance ofsymptoms in smokers with preserved pulmonary function. N Engl JMed. 2016;374:1811-21.

12. Martinez FJ, Han MLK, Allinson JP, et al. At the Root: Defining and Halting Progression of Early Chronic Obstructive Pulmonary Disease. Am J Respir Crit Care Med Vol. 2018;197(12):1540-51.

13. Agusti A, Faner R. How to Define Early COPD, the American Thoracic Society. 2018;(5).

14. Kirby M, Tanabe N, Tan WC, et al. Total Airway Count on Computed Tomography and the Risk of Chronic Obstructive Pulmonary Disease Progression. American Journal of Respiratory Critical Care Medicine Volume. 2018;1(1):56-65.

15. Bhatt SP, Bhakta NR, Wilson CG, et al. New Spirometry Indices for Detecting Mild Airflow Obstruction. Scientific Report. 2018;(8).

16. Li Y, Dai YL, Yu N, et al. Sex-related differences in bronchial parameters and pulmonary function test results in patients with chronic obstructive pulmonary disease based on three-dimensional quantitative computed tomography. J Int Med Res. 2018;46(1):135-42.

17. Li Y, Dai YL, Yu N, et al. Sex-related differences in bronchial parameters and pulmonary function test results in patients with chronic obstructive pulmonary disease based on three-dimensional quantitative computed tomography. $\mathrm{J}$ Int Med Res. 2018;46(1):135-42.

18. Hogg JC, Chu F, Utokaparch S, et al. The nature of small-airway obstruction in chronic obstructive pulmonary disease. $\mathrm{N}$ Engl J Med. 2004;350(26):2645-53.

19. Li Y, Dai YL, Yu N, et al. Sex-related differences in bronchial parameters and pulmonary function test results in patients with chronic obstructive pulmonary disease based on three-dimensional quantitative computed tomography. $\mathrm{J}$ Int Med Res. 2018;46(1):135-42.

20. Xia GQ, Chang Q, Lu XM, et al. Discussion on the clinical significance of small airway function index examination in patients with COPD. Medical Information. 2016;5(29).

21. Vasilescu D, Marchetti N, Galban CJ, et al. The relationship between functional small airways disease and small airways pathology in COPD. Am J Respir Crit Care Med. 2017;195:5157.

22. Boes JL, Hoff BA, Bule M, et al. Parametric response mapping monitors temporal changes on lung CT scans in the subpopulations and intermediate outcome measures in COPD Study (SPIROMICS). Acad Radiol. 2015;22:186-94.

23. Harvey BG, Strulovici-Barel Y, Kaner RJ, et al. Risk of COPD with obstruction in active smokers with normal spirometry and reduced diffusion capacity. Eur Respir J. 2015;46:1589-96.

\section{Tables}

\section{Table 1}

Correlations between digital lung 3D imaging technology parameters and PFT

Page 8/13 


\begin{tabular}{|c|c|c|c|c|c|c|c|c|c|c|}
\hline \multirow{2}{*}{\multicolumn{2}{|c|}{ Project }} & FEV1 & FEV1\% & FEV1 & & & & & & \\
\hline & & & & /FVC & $\mathrm{O}(\%)$ & $\begin{array}{l}25- \\
75\end{array}$ & (L/sec) & (L/sec) & (L/sec) & (L/sec) \\
\hline \multicolumn{2}{|l|}{ LV (L) } & $-0.319^{\star \star}$ & $-0.305^{\star \star}$ & $-0.516^{* *}$ & $-0.181^{*}$ & $-0.467^{\star \star}$ & $-0.512^{\star \star}$ & $-0.520^{\star \star}$ & $-0.486^{\star *}$ & $-0.425^{\star *}$ \\
\hline \multicolumn{2}{|l|}{ \%LAA950 } & $-0.445^{\star \star}$ & $-0.447^{\star \star}$ & $-0.645^{\star \star}$ & $-0.172^{*}$ & $-0.420^{\star \star}$ & $-0.646^{\star \star}$ & $-0.691^{\star \star}$ & $-0.661^{\star \star}$ & $-0.549^{\star *}$ \\
\hline \multicolumn{2}{|c|}{ PRM fsad (\%) } & $-0.368^{\star \star}$ & $-0.371^{\star *}$ & $-0.611^{\star *}$ & -0.163 & $-0.555^{\star \star}$ & $-0.560^{\star \star}$ & $-0.587^{\star \star}$ & $-0.574^{\star *}$ & $-0.455^{\star *}$ \\
\hline \multicolumn{2}{|c|}{ PRM Emph (\%) } & $-0.46^{* *}$ & $-0.424^{* *}$ & $-0.614^{\star *}$ & $-0.190^{*}$ & $-0.641^{\star \star}$ & $-0.646^{\star *}$ & $-0.680^{\star \star}$ & $-0.648^{* *}$ & $-0.542^{* *}$ \\
\hline \multirow[t]{5}{*}{$\begin{array}{l}\text { Generation } \\
5\end{array}$} & $\begin{array}{l}\text { LD } \\
(\mathrm{mm})\end{array}$ & $0.394^{\star \star}$ & $0.368^{\star \star}$ & $0.532^{\star \star}$ & $0.305^{\star \star}$ & $0.556^{\star \star}$ & $0.576^{\star \star}$ & $0.564^{\star \star}$ & $0.505^{\star \star}$ & $0.438^{\star \star}$ \\
\hline & $\begin{array}{l}\text { WT } \\
(\mathrm{mm})\end{array}$ & $-0.279^{*}$ & $-0.275^{*}$ & $-0.569^{\star \star}$ & -0.044 & $-0.473^{\star \star}$ & $-0.419^{\star *}$ & $-0.262^{*}$ & $-0.494^{\star \star}$ & $-0.364^{\star *}$ \\
\hline & $\begin{array}{l}\mathrm{LA} \\
\left(\mathrm{mm}^{2}\right)\end{array}$ & $0.315^{\star \star}$ & $0.437^{\star \star}$ & $0.539^{\star \star}$ & $0.263^{*}$ & $0.233^{*}$ & $0.484^{\star *}$ & $0.507^{\star \star}$ & $0.470^{\star \star}$ & $0.375^{\star \star}$ \\
\hline & WA\% & $-0.353^{\star \star}$ & $-0.402^{\star \star}$ & $-0.644^{\star \star}$ & $-0.188^{\star}$ & $-0.584^{\star \star}$ & $-0.605^{\star \star}$ & $-0.616^{\star \star}$ & $-0.538^{\star \star}$ & $-0.382^{\star \star}$ \\
\hline & $\begin{array}{l}\text { TDR } \\
(\%)\end{array}$ & $-0.322^{\star \star}$ & $-0.343^{\star *}$ & $-0.558^{\star \star}$ & $-0.182^{*}$ & $-0.538^{* \star}$ & $-0.574^{\star *}$ & $-0.566^{\star *}$ & $-0.483^{* \star}$ & $-0.378^{\star \star}$ \\
\hline \multirow[t]{5}{*}{$\begin{array}{l}\text { Generation } \\
6\end{array}$} & $\begin{array}{l}\text { LD } \\
(\mathrm{mm})\end{array}$ & $0.387^{\star \star}$ & $0.377^{\star \star}$ & $0.628^{\star \star}$ & $0.191^{\star}$ & $0.556^{\star \star}$ & $0.594^{\star *}$ & $0.601^{\star *}$ & $0.578^{\star \star}$ & $0.443^{\star *}$ \\
\hline & $\begin{array}{l}\text { WT } \\
(\mathrm{mm})\end{array}$ & $-0.287^{\star}$ & $-0.237^{\star}$ & $-0.472^{\star \star}$ & -0.068 & $-0.447^{\star \star}$ & $-0.466^{\star \star}$ & $-0.485^{\star \star}$ & $-0.457^{\star \star}$ & $-0.392^{\star \star}$ \\
\hline & $\begin{array}{l}\mathrm{LA} \\
\left(\mathrm{mm}^{2}\right)\end{array}$ & $0.318^{\star \star}$ & $0.346^{\star *}$ & $0.631^{\star \star}$ & $0.224^{\star}$ & $0.545^{\star *}$ & $0.571^{\star \star}$ & $0.605^{\star \star}$ & $0.528^{\star \star}$ & $0.400^{\star \star}$ \\
\hline & WA\% & $-0.404^{\star \star}$ & $-0.479^{\star \star}$ & $-0.664^{\star *}$ & $-0.252^{\star}$ & $-0.630^{\star \star}$ & $-0.623^{\star \star}$ & $-0.692^{\star \star}$ & $-0.616^{\star *}$ & $-0.460^{\star *}$ \\
\hline & $\begin{array}{l}\text { TDR } \\
(\%)\end{array}$ & $-0.350^{\star \star}$ & $-0.370^{\star \star}$ & $-0.606^{\star \star}$ & $-0.171^{\star}$ & $-0.559^{\star \star}$ & $-0.578^{\star \star}$ & $-0.619^{\star \star}$ & $-0.540^{\star \star}$ & $-0.444^{\star \star}$ \\
\hline \multirow[t]{5}{*}{$\begin{array}{l}\text { Generation } \\
7\end{array}$} & $\begin{array}{l}\text { LD } \\
(\mathrm{mm})\end{array}$ & $0.398^{\star \star}$ & $0.456^{\star \star}$ & $0.624^{\star \star}$ & $0.277^{\star}$ & $0.569^{\star \star}$ & $0.584^{\star \star}$ & $0.607^{\star \star}$ & $0.583^{\star \star}$ & $0.483^{\star \star}$ \\
\hline & $\begin{array}{l}\text { WT } \\
(\mathrm{mm})\end{array}$ & $-0.379^{\star \star}$ & $-0.349^{\star \star}$ & $-0.592^{\star \star}$ & -0.140 & $-0.533^{\star \star}$ & $-0.578^{\star \star}$ & $-0.607^{\star \star}$ & $-0.552^{\star \star}$ & $-0.447^{\star *}$ \\
\hline & $\begin{array}{l}\mathrm{LA} \\
\left(\mathrm{mm}^{2}\right)\end{array}$ & $0.426^{\star \star}$ & $0.385^{\star *}$ & $0.601^{\star *}$ & $0.228^{\star}$ & $0.583^{* *}$ & $0.617^{\star \star}$ & $0.632^{\star \star}$ & $0.571^{\star *}$ & $0.474^{* *}$ \\
\hline & WA\% & $-0.344^{\star \star}$ & $-0.421^{\star *}$ & $-0.664^{\star \star}$ & $-0.168^{\star}$ & $-0.580^{\star \star}$ & $-0.604^{\star \star}$ & $-0.643^{\star \star}$ & $-0.558^{\star \star}$ & $-0.416^{\star *}$ \\
\hline & $\begin{array}{l}\text { TDR } \\
(\%)\end{array}$ & $-0.296^{*}$ & $-0.350^{\star \star}$ & $-0.608^{\star \star}$ & -0.090 & $-0.512^{\star \star}$ & $-0.521^{\star \star}$ & $-0.581^{\star \star}$ & $-0.495^{\star \star}$ & $-0.388^{\star *}$ \\
\hline
\end{tabular}

Note: ${ }^{\star} P<0.05$, which is relevant; ${ }^{* \star} P<0.01$, which is clearly correlated.

\section{Table 2}

Comparisons of PFT and digital lung 3D imaging technology parameters between two groups 


\begin{tabular}{|c|c|c|c|c|}
\hline \multicolumn{2}{|l|}{ Project } & Observation group & $\begin{array}{l}\text { Control } \\
\text { group }\end{array}$ & $P$ \\
\hline \multicolumn{5}{|l|}{ Demographics } \\
\hline \multicolumn{2}{|l|}{ Age (yrs) } & $50 \pm 3$ & $52 \pm 5$ & $>0.05$ \\
\hline \multicolumn{2}{|c|}{ Sex ratio (male/female) } & $46 / 44$ & $23 / 23$ & $>0.05$ \\
\hline \multicolumn{2}{|c|}{ Smoking composition ratio (yes/no) } & $47 / 43$ & $22 / 24$ & $>0.05$ \\
\hline \multicolumn{2}{|l|}{ BMI $\left(\mathrm{kg} / \mathrm{m}^{2}\right)$} & 27.10 & 26.90 & $>0.05$ \\
\hline \multicolumn{5}{|l|}{ PFT } \\
\hline \multicolumn{2}{|l|}{ FEV1 (L) } & $2.24 \pm 0.62$ & $2.97 \pm 0.66$ & $<0.001$ \\
\hline \multicolumn{2}{|l|}{ FEV1\% } & $80.79 \pm 12.42$ & $94.00 \pm 8.58$ & $<0.001$ \\
\hline \multicolumn{2}{|l|}{ FEV1/FVC } & $75.00 \pm 5.24$ & $84.75 \pm 3.85$ & $<0.001$ \\
\hline \multicolumn{2}{|l|}{ MEF25-75 (L/sec) } & $1.77 \pm 0.53$ & $3.47 \pm 1.23$ & $<0.001$ \\
\hline \multicolumn{2}{|l|}{ MEF25 (L/sec) } & $0.80 \pm 0.30$ & $1.53 \pm 0.42$ & $<0.001$ \\
\hline \multicolumn{2}{|l|}{ MEF50 (L/sec) } & $2.20 \pm 0.66$ & $4.00 \pm 0.94$ & $<0.001$ \\
\hline \multicolumn{2}{|l|}{ MEF75 (L/sec) } & $4.31 \pm 1.18$ & $6.95 \pm 1.50$ & $<0.001$ \\
\hline \multicolumn{2}{|l|}{ PEF (L/sec) } & $5.43 \pm 1.64$ & $7.86 \pm 1.74$ & $<0.001$ \\
\hline \multicolumn{2}{|l|}{ DLCO (\%) } & $82.30 \pm 6.66$ & $86.72 \pm 12.34$ & 0.007 \\
\hline \multicolumn{5}{|l|}{ 3D-CT } \\
\hline \multicolumn{2}{|l|}{ LV (L) } & $5.61 \pm 0.80$ & $4.87 \pm 0.42$ & $<0.001$ \\
\hline \multicolumn{2}{|l|}{$\%$ LAA950 } & $5.23 \pm 0.74$ & $3.18 \pm 0.43$ & $<0.001$ \\
\hline \multicolumn{2}{|l|}{ PRM fsad(\%) } & $17.86 \pm 2.00$ & $11.38 \pm 2.86$ & $<0.001$ \\
\hline \multicolumn{2}{|l|}{ PRM Emph(\%) } & $0.59 \pm 0.13$ & $0.06 \pm 0.06$ & $<0.001$ \\
\hline \multirow[t]{5}{*}{ Generation 5} & LD (mm) & $4.04 \pm 0.07$ & $4.30 \pm 0.12$ & $<0.001$ \\
\hline & WT (mm) & $1.55 \pm 0.12$ & $1.26 \pm 0.12$ & $<0.001$ \\
\hline & $\mathrm{LA}\left(\mathrm{mm}^{2}\right)$ & $11.36 \pm 0.95$ & $14.80 \pm 1.79$ & $<0.001$ \\
\hline & WA\% & $69.04 \pm 2.01$ & $61.79 \pm 3.01$ & $<0.001$ \\
\hline & TDR (\%) & $22.69 \pm 1.40$ & $18.90 \pm 2.11$ & $<0.001$ \\
\hline \multirow[t]{5}{*}{ Generation 6} & $\mathrm{LD}(\mathrm{mm})$ & $3.00 \pm 0.08$ & $3.28 \pm 0.11$ & $<0.001$ \\
\hline & $\mathrm{WT}(\mathrm{mm})$ & $1.52 \pm 0.22$ & $1.20 \pm 0.09$ & $<0.001$ \\
\hline & $\mathrm{LA}\left(\mathrm{mm}^{2}\right)$ & $6.30+0.79$ & $9.09 \pm 0.81$ & $<0.001$ \\
\hline & WA\% & $70.53 \pm 1.75$ & $62.87 \pm 2.59$ & $<0.001$ \\
\hline & TDR (\%) & $23.52 \pm 1.37$ & $19.76 \pm 1.54$ & $<0.001$ \\
\hline \multirow[t]{2}{*}{ Generation 7} & $\mathrm{LD}(\mathrm{mm})$ & $2.02 \pm 0.06$ & $2.26 \pm 0.11$ & $<0.001$ \\
\hline & $\mathrm{WT}(\mathrm{mm})$ & $1.21 \pm 0.09$ & $0.98 \pm 0.07$ & $<0.001$ \\
\hline
\end{tabular}

Page 10/13 


\begin{tabular}{|llll|}
\hline LA $\left(\mathrm{mm}^{2}\right)$ & $3.22 \pm 0.61$ & $5.47 \pm 0.71$ & $<0.001$ \\
\hline WA\% & $70.70 \pm 1.88$ & $63.71 \pm 2.86$ & $<0.001$ \\
\hline TDR $(\%)$ & $24.03 \pm 1.74$ & $20.12 \pm 1.67$ & $<0.001$ \\
\hline
\end{tabular}

\section{Figures}
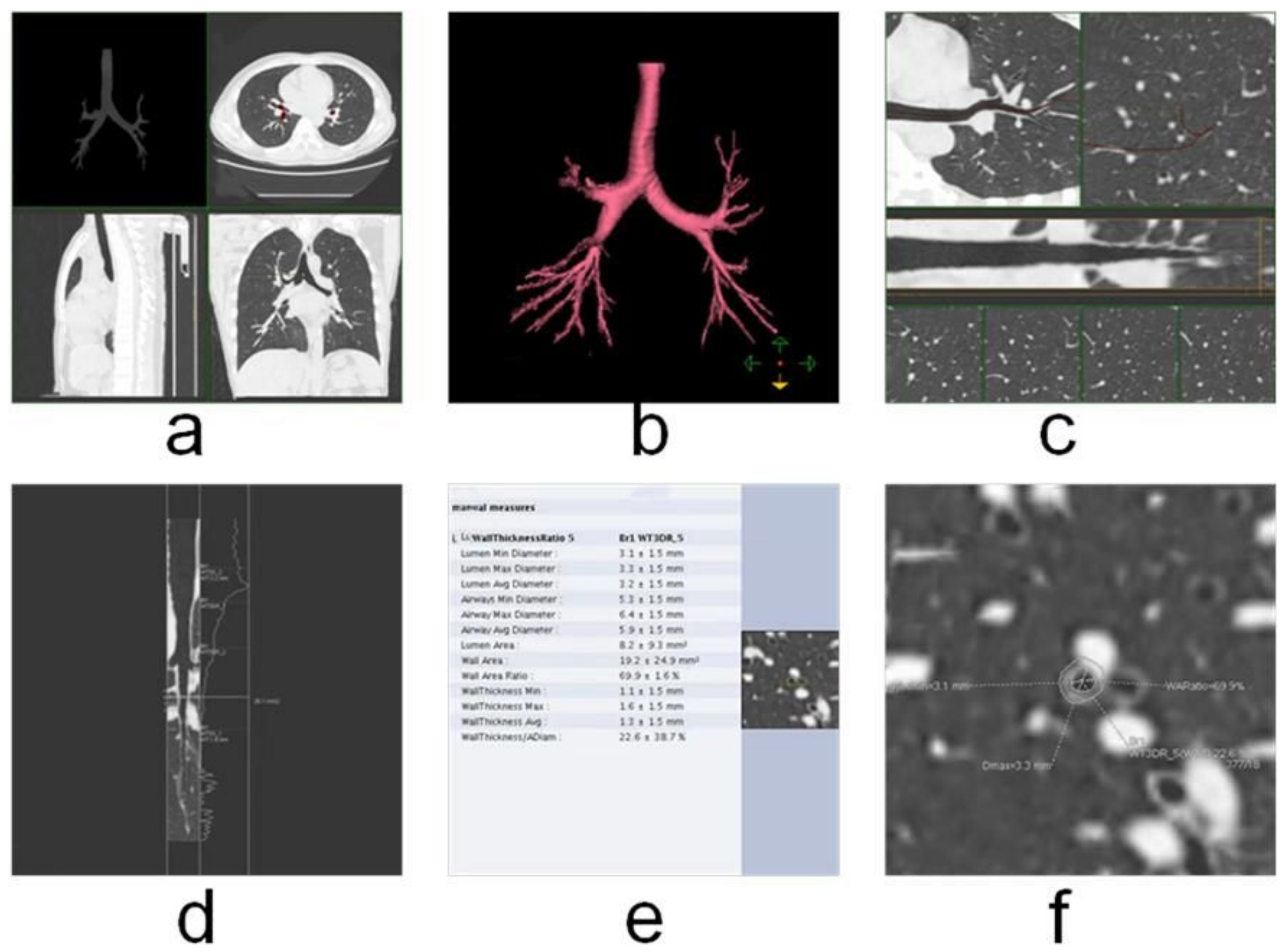

Figure 1

post-processing software segmentation of the respiratory biphasic bronchial tree (a-b), to obtain the measurement position of generations 5 to 7 bronchus (c-f). 


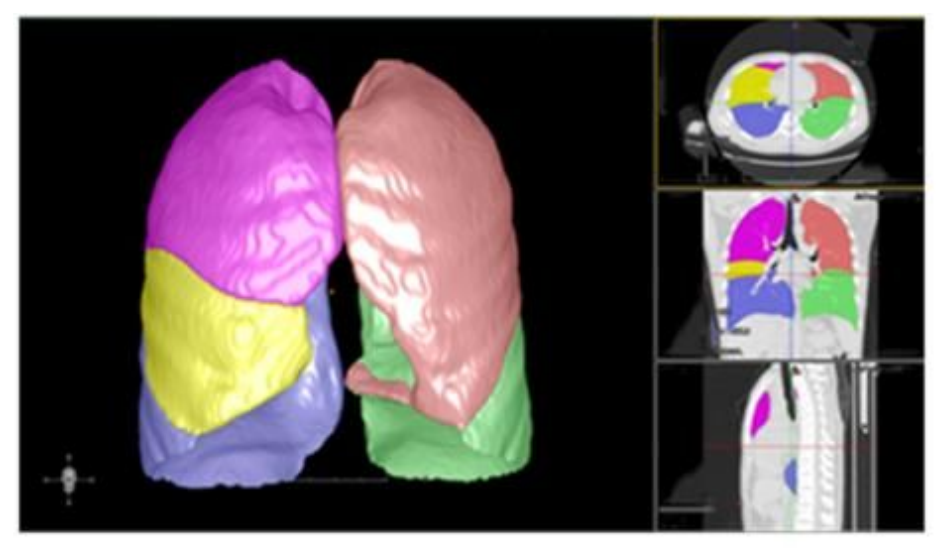

a

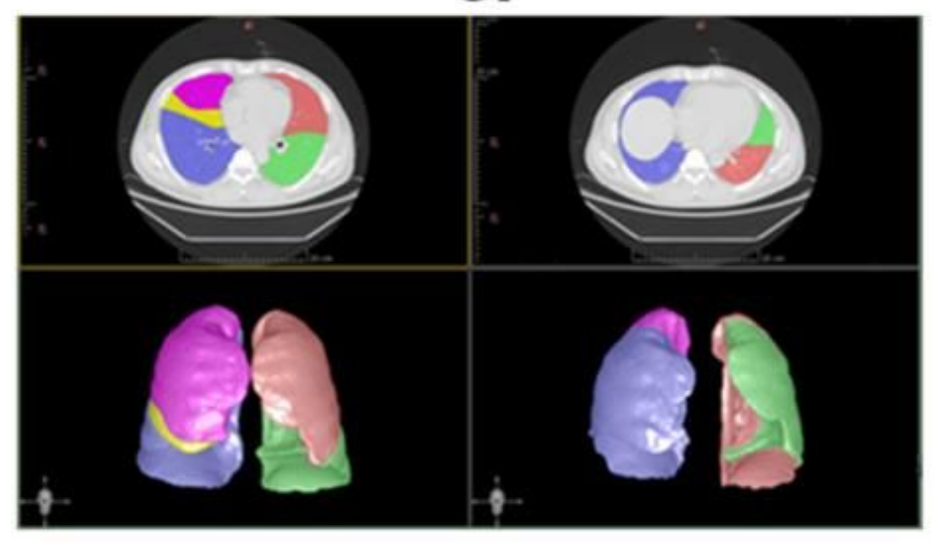

C

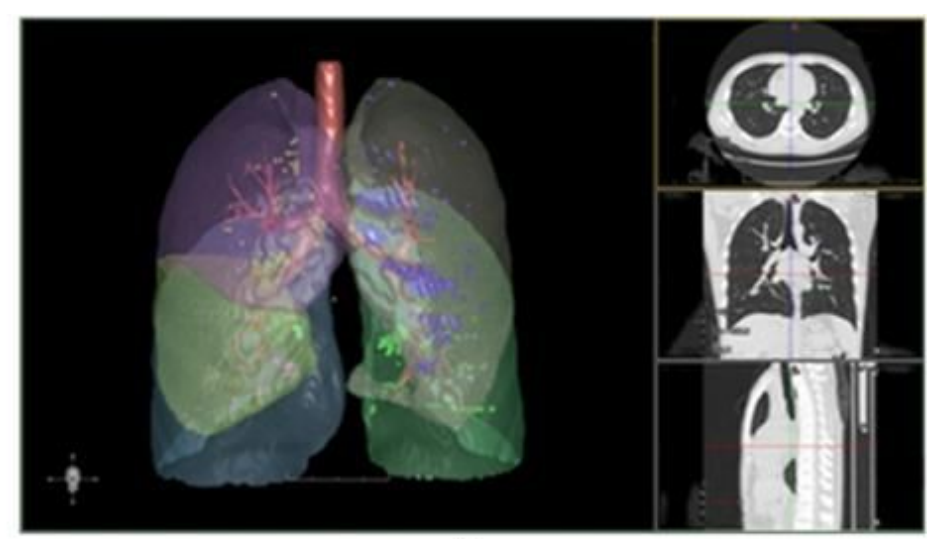

b

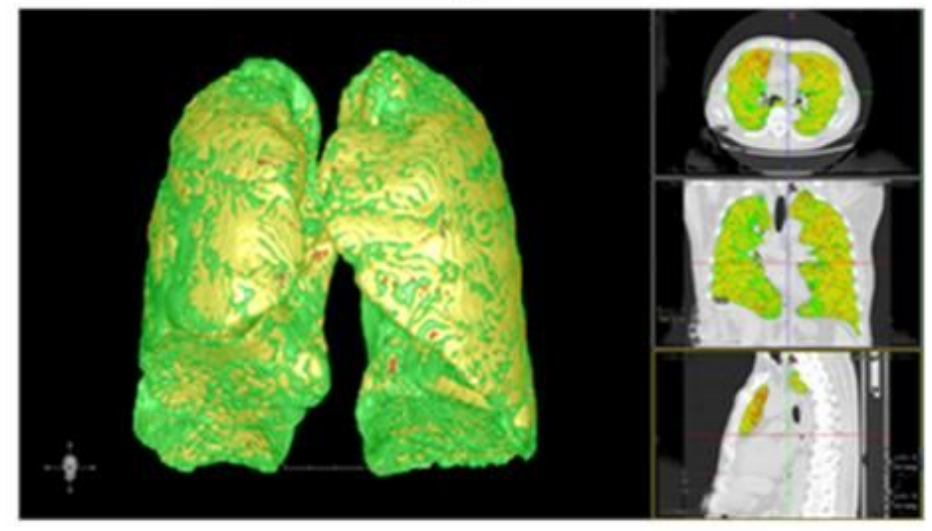

d

Figure 2

the operation diagram of PRM fSAD, PRM Emph, LV, \%LAA950 (a-d). 


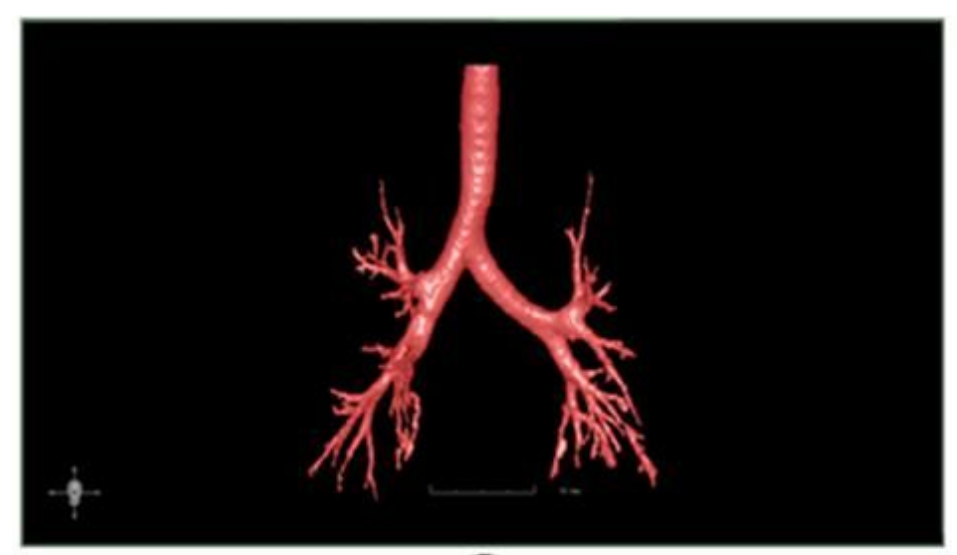

a

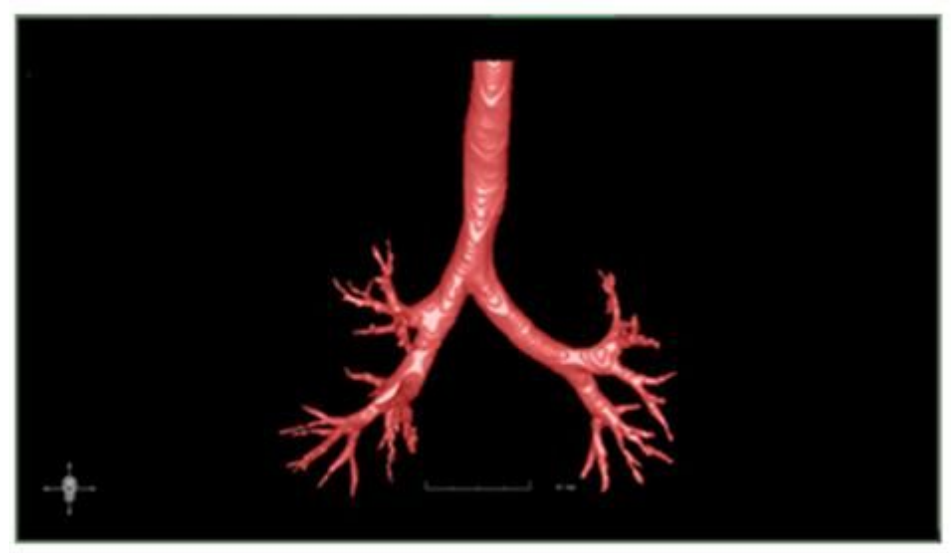

C

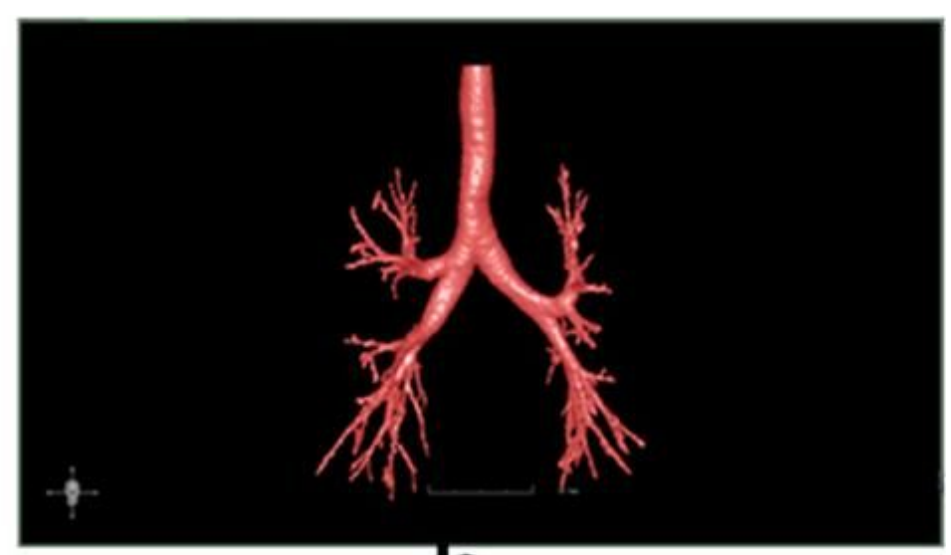

b

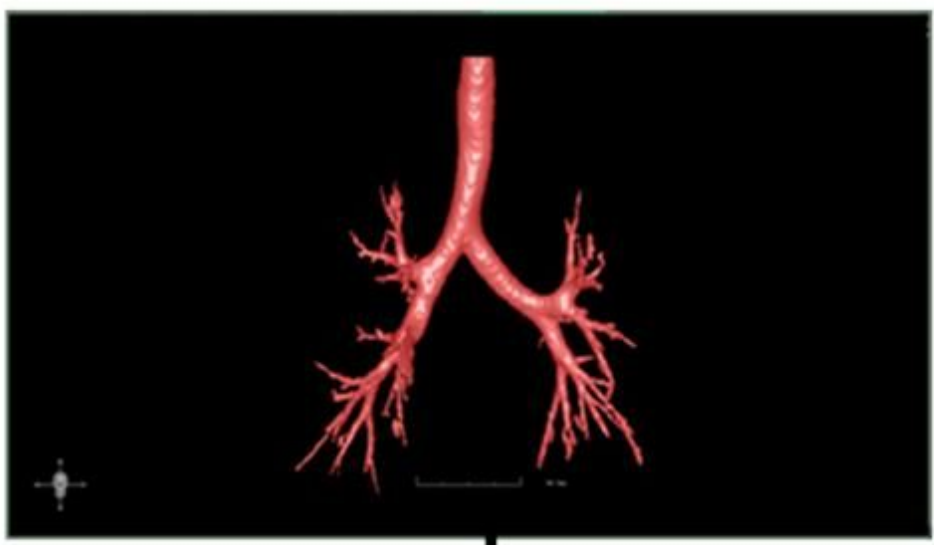

d

\section{Figure 3}

expiratory bronchial tree in the control group (a), inspiratory bronchial tree in the control group (b), expiratory bronchial tree in the observation group (c), inspiratory bronchial tree in the observation group (d). 